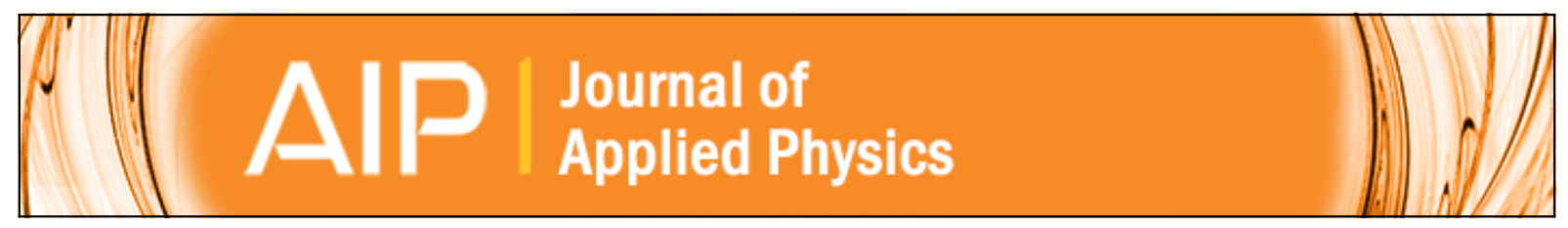

\title{
Semiclassical electronic transport calculations in multilayered granular alloys
}

J. Milano and A. M. Llois

Citation: Journal of Applied Physics 102, 013705 (2007); doi: 10.1063/1.2751083

View online: http://dx.doi.org/10.1063/1.2751083

View Table of Contents: http://scitation.aip.org/content/aip/journal/jap/102/1?ver=pdfcov

Published by the AIP Publishing

Articles you may be interested in

Validation of the tunneling percolation staircase model in granular metals

Appl. Phys. Lett. 104, 253109 (2014); 10.1063/1.4885498

Conductivity and scaling properties of chemically grown granular silver films

J. Appl. Phys. 106, 063722 (2009); 10.1063/1.3226859

Magnetic and transport properties of $\mathrm{Ag} / \mathrm{Co} 90 \mathrm{Fe} 10$ granular multilayers

J. Appl. Phys. 96, 7392 (2004); 10.1063/1.1810638

Electronic structure of $\operatorname{InN} x$ As 1-x alloys from tight-binding calculations

Appl. Phys. Lett. 76, 3576 (2000); 10.1063/1.126711

Ferromagnetic resonance in granular thin films

J. Appl. Phys. 85, 5654 (1999); 10.1063/1.369830

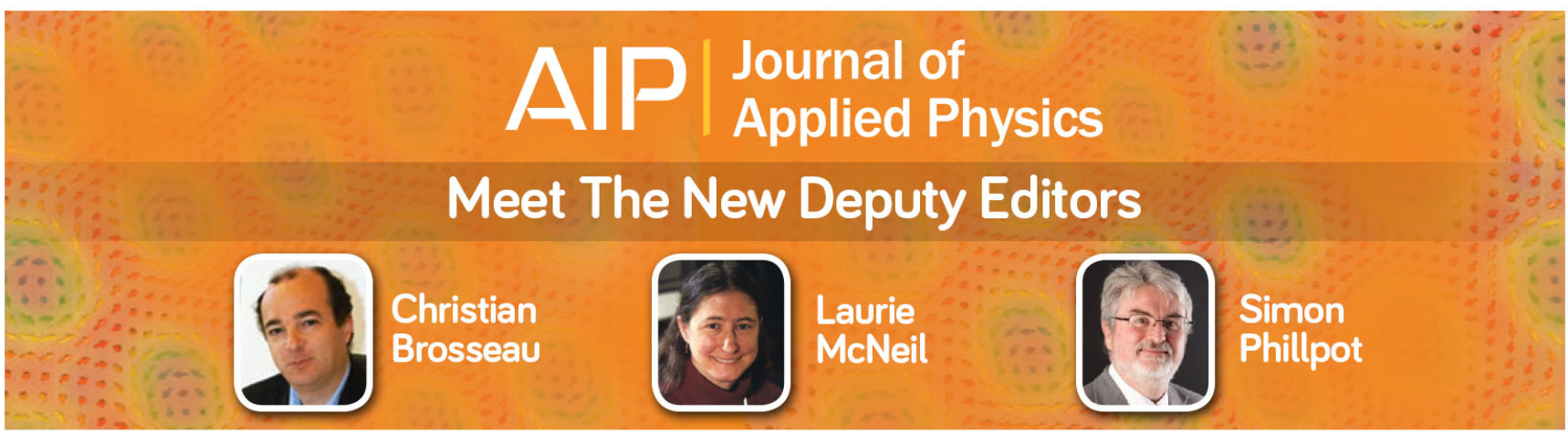




\title{
Semiclassical electronic transport calculations in multilayered granular alloys
}

\author{
J. Milano ${ }^{a)}$ \\ Departamento de Física, Comisión Nacional de Energía Atómica, San Martín, Argentina \\ A. M. Llois \\ Departamento de Física, Comisión Nacional de Energía Atómica, San Martín, Argentina \\ and Departamento de Física "Juan José Giambiagi", Facultad de Ciencias Exactas y Naturales, \\ Universidad de Buenos Aires, Buenos Aires, Argentina
}

(Received 30 October 2006; accepted 21 May 2007; published online 6 July 2007)

\begin{abstract}
We have calculated the electrical conductivity in the current-in-plane geometry of multilayered granular alloys composed of Co clusters embedded in Ag alternating with pure Ag layers. In particular, we have paid attention to the conductivity behavior as a function of Ag layer thickness, Co clusters' size, and degree of percolation. The electronic structure is self-consistently calculated within the unrestricted Hartree-Fock approximation using a parametrized tight binding Hamiltonian which includes a Hubbard-like term. The conductivity tensor is obtained by using the semiclassical Boltzmann equation in the anisotropic relaxation time approximation. We have used a $s-d$ Mott-like scattering model for the electronic mean free path taking into account the Sondheimer's picture for electronic transport in thin films. We find that the experimental conductivity behavior at coalescence can be explained through the electronic band contribution. The conductivity behavior of continuous multilayers is already attained in the very early stage of percolation, as in the experiments. (C) 2007 American Institute of Physics. [DOI: 10.1063/1.2751083]
\end{abstract}

\section{INTRODUCTION}

The discovery of giant magnetoresistance (GMR) in metallic magnetic multilayers ${ }^{1}$ (MMMLs), as well as in metallic magnetic granular alloys ${ }^{2}$ (MMGAs), has strengthened the research activity on transport properties in the last decade. However, it has not yet been easy to obtain a suitable theoretical model to describe the electrical transport in MMGAs due to the random arrangement and shape of the clusters within the samples and because of their size distribution.

Most of the conductivity models appearing in the literature are based on the comparison of the transport behavior between MMGAs and MMMLs. The first model for MMGAs was proposed by Zhang and Levy, ${ }^{3}$ in which they state that transport in MMGAs behaves mostly as CPP transport (electric current flowing perpendicular to the layers) in MMMLs. This means that the resistivity arises mainly from scattering of the conduction electrons with the surface of the magnetic clusters and this is proportional to the total cluster's area facing the transport direction. This model should lead to a monotonous increment of the GMR as the clusters' radii are reduced, at constant concentration of the magnetic atoms building the clusters. However, this effect is not experimentally observed because the GMR reaches its maximum value for a given cluster radius ${ }^{2}$ and for smaller radii it decreases. To explain this decrease, Shen et al. ${ }^{4}$ proposed that it is due to the fact that the conduction electrons bypass the very small clusters and that, thereafter, the contribution of the spin-dependent scattering at the interfaces goes down. Ferrari et al., ${ }^{5}$ using classical electrodynamics of continuous

\footnotetext{
a) Present address: CNEA-Centro Atómico Bariloche, (R8402AGP) San Carlos de Bariloche, Argentina; electronic-mail: milano@cab.cnea.gov.ar
}

media, proposed that the transport behavior in MMGAs is a mixture of both transport geometries in MMMLs, CIP (current flowing parallel to layers) and CPP. In particular, the CPP-like regime can be achieved, within Ferrari's model, when the conductivity of the granules is much larger than the metallic matrix's one.

In order to satisfy technological requirements Holody et al. ${ }^{6}$ introduced the study of discontinuous magnetic multilayers, being the first ones to measure the transport properties of these type of multilayers, namely, of $\mathrm{Co} / \mathrm{Ag} / \mathrm{NiFe} / \mathrm{Ag}$ multilayered systems. In these systems the Co layers are discontinuous as the amount of deposited Co is not enough to form complete layers. Later on, several experimental works were published on this kind of systems ${ }^{7-9}$ with the aim of explaining their transport and magnetic properties, however, a thorough theoretical understanding is still lacking due to the difficulties that arise if one wants to treat, on equal footing, the high conductivity regions as well as the granular ones. Moreover, from a basic understanding, granular magnetic multilayers constitute ideal alloys to study the evolution of the magnetic and transport properties when going from a granular alloy to a multilayered granular one and from this intermediate situation, through a topological transition, to a multilayer with continuous layers.

In a previous work we showed that in granular multilayers the Ag layer thickness, $t_{\mathrm{Ag}}$, plays an important role in the onset of this transition. ${ }^{8}$ We found that the scattering behavior in the granular layers depends on $t_{\mathrm{Ag}}$. For small $t_{\mathrm{Ag}}$ values, the clusters behave as scattering centers in a similar way as they do in MMGAs. For large $t_{\mathrm{Ag}}$ values their effect is just to reduce the electronic mean free path. We also showed that in granular multilayers the conductivity behavior corre- 
sponding to a multilayer with continuous magnetic layers is reached at the very first stage of percolation onset.

Based on experimental facts, the aim of this work is to address the behavior of the CIP resistivity along transitions from (a) multilayered granular alloys to multilayers with continuous magnetic layers and from (b) multilayered granular alloys to MMGAs. For the calculations, we consider multilayers of the type $\left[\mathrm{Co}_{1-x} \mathrm{Ag}_{x}\right] / \mathrm{Ag}$ as they belong to the systems which are more thoroughly experimentally studied nowadays. In order to obtain the conductivity tensor, the transport calculations are done within the semiclassical Boltzmann approximation. The band contribution to transport is explicitly taken into account through the semiclassical velocities which enter into the linearized Boltzmann equation. The relaxation time is taken to be $\mathbf{k}$ and spin dependent (anisotropic) as it is explained in Sec. II. The scattering processes at the clusters' surfaces are taken into account through a Mott-like model for the relaxation time, ${ }^{10}$ which is well suited for diffusive scattering, as it is the case in our systems. We also take explicitly into account the short circuit through the pure silver layers which takes place when the thickness of these layers is large enough and for this we employ the Sondheimer picture. ${ }^{11}$

The semiclassical Boltzmann approximation to obtain the conductivity tensor has been successfully applied by us to magnetic multilayers with continuous layers in previous work. $^{12}$

\section{MODEL}

Multilayered granular alloys present, both, random order and periodicity. The magnetic clusters are randomly distributed within layers which alternate periodically with the conducting layers. These two characteristic features, namely, the granular one and the periodic multilayered stacking on the other, define the interplay between two contributions to transport, which are intertwined due to the diffusive motion of the carriers. On the one hand, the electrons find low resistivity paths when traveling within the pure Ag layers (Agls); on the other hand, the electrons that enter into the granular layers (Cols) undergo spin dependent scattering at the interfaces and inside the clusters. We propose a model which takes into account the two mentioned transport contributions. The Boltzmann equation is a suitable scheme for treating our problem, because it allows us to model, both, the high conductivity contribution due to the Ag layers and the spin dependent scattering at the Co clusters via band velocities and relaxation time respectively.

The fact that the resistivity is mainly given by scattering at the clusters' interfaces and that the contributions due to magnons and phonons can be disregarded, as is explained later, makes it important to briefly discuss the role of weak localization $^{13}$ on the transport properties of our systems. Microscopically, weak localization sets in when the phase relaxation length is greater than the mean free path. If this condition is fulfilled, the phase coherence leads the conductivity to be sensitive to the arrangement of the scattering centers due to quantum interference. On the other hand, phase coherence is destroyed by dynamic scattering, i.e., phonons, magnons. In addition, scattering at magnetic centers, like our Co clusters, also destroys phase coherence due to spin fluctuation. This indicates that the phase relaxation length is of the same order as the mean free path and, thereafter, weak localization effects are negligible. Moreover, from a macroscopic point of view, the sample can be seen as an ensemble of phase coherent units. If we consider large sample sizes, the effect of weak localization is smoothed due to averaging of the statistical fluctuations over the phase coherent units. Therefore, due to microscopic and macroscopic reasons, we consider it reasonable to neglect contributions due to weak localization effects.

The conductivity is obtained from the Boltzmann equation in the anisotropic relaxation time approximation, no spin-flip scattering is considered. ${ }^{14}$ The conductivity tensor (or resistivity) within this approximation is then given by

$$
\frac{1}{\rho_{i i}}=\sigma_{i i}=\frac{e^{2}}{8 \pi^{2}} \sum_{n s} \int \tau_{n}^{s}(\mathbf{k}) v_{i n}^{s}(\mathbf{k}) v_{i n}^{s}(\mathbf{k}) \delta\left[\varepsilon_{n}^{s}(\mathbf{k})-\varepsilon_{F}\right] d^{3} \mathbf{k},
$$

where $s$ denotes the spin index, $n$ is the band index, and $\varepsilon_{F}$ is the Fermi energy. To obtain the semiclassical velocities, $v_{i n}^{s}(\mathbf{k})$, the electronic bands are used. $\tau_{n}^{s}$ is the relaxation time and it is assumed to be dependent on spin and $\mathbf{k}$, as it is explained later. Within a semiclassical picture, the contribution to transport arising from the band structure, that is from the semiclassical velocities, contains information on the periodic multilayered arrangement as well as on the degree of Co-Ag band hybridization at the surface of the clusters. Using this formalism, we have obtained very good results for $\mathrm{Fe} / \mathrm{Cu}$ metallic multilayers doped with $\mathrm{Cr}^{12}$ Each system for which we obtain the band contribution is periodic and contains clusters of a given size, namely, no dispersion in the cluster sizes is considered in the band structure calculations. The scattering effects and noncoherent contributions due to the nonperiodic arrangement of the clusters are taken into account in the mean free paths used to evaluate the relaxation times.

Due to the high effective mass of the electrons scattered into the clusters' Co $d$ states, their contribution to the resistivity is more important than the one corresponding to $s$ states. Originally, Mott ${ }^{10}$ used this idea to explain the anomalous behavior of the resistivity in disordered magnetic AgPd alloys. In Mott's model scattering into $d$ states arises from random collisions with phonons. In our case, scattering is due to the collisions with the randomly distributed Co clusters. We consider that the transport regime is completely diffusive and this is taken into account in the model for the mean free path.

We derive the expression for the mean free path assuming a Mott-like scattering in the granular multilayers by considering that the electrons travel mainly within Agls until they scatter with Cols. Three different kinds of scattering events can be considered: (i) electrons can be scattered to a Co $d$ state, (ii) they can be scattered to another $s$ state or, (iii) they can suffer no scattering. Processes of type (ii) include backscattering and specular scattering at interfaces. Backscattering processes are important in CPP geometry and 
many-body vertex corrections ${ }^{15}$ should be taken into account for a correct description. In CIP, the geometry in which we are working, backscattering does not play a decisive role and it will be neglected. Specular scattering at the interfaces is important in CIP, but due to the random arrangement of the clusters within the granular layers, it can be addressed to as a noncoherent scattering contribution as it has been already pointed out earlier.

The contribution to the mean free path due to scattering at the granular layers depends on the electron motion perpendicular to them. We focus then on the component of the mean free path perpendicular to the layers, $\ell$. To calculate $\ell$ we consider only scattering processes into localized $d$ states. The effect on the conductivity due to scattering into $s$ states is already considered through the effective mean free path of the pure $\mathrm{Ag}$ layers, $\lambda_{\mathrm{Ag}}$. The electrons which are scattered to a $d$ state no longer contribute to the conduction, whereas the fraction which is not being scattered keeps on traveling up to the next Col. Due to disorder in the granular layers the scattering processes in subsequent layers are totally independent, thereafter the mechanism at the next $\mathrm{Col}$ is the same as in the previous one.

The transverse component of the electronic mean free path, $\ell$, is obtained by summing up the perpendicular distances traveled by the electrons weighted by the fraction of electrons which suffer no $d$ scattering. We propose, then, the following expression:

$$
\ell^{s}=t_{\mathrm{Ag}} \frac{\sum_{i=0}^{\infty} i\left(1-r^{s}\right)^{i}}{\sum_{i=0}^{\infty}\left(1-r^{s}\right)^{i}}=\frac{t_{\mathrm{Ag}}}{r^{s}},
$$

where $t_{\mathrm{Ag}}$ is the thickness of the Agls, $s$ is the spin direction, and $r$ is the fraction of electrons that are scattered within the $i$ th Col. Within our model, the scattering rate to localized $d$ states depends on the relative amount of $d$ states on the Fermi surface, then $r=N_{d} / N$, where $N_{d}$ is the density of Co $d$ states and $N$ is the total density of states, both evaluated at the Fermi surface. The denominator is a normalization factor.

From the last expression it is seen that if $r$ is close to one, $\ell^{s}$ tends to $t_{\mathrm{Ag}}$ indicating that for those values of $r$ the electrons are scattered strongly in the first granular layers. The opposite situation is given if $N_{d}$ is too small and the mean free path becomes larger than $\lambda_{\mathrm{Ag}}$. The electrons for which the last situation occurs are not taken into account when evaluating Eq. (2), as it was proposed by Sondheimer. ${ }^{11}$ In this case the interfacial effects (in our case due to the granular layer) over the mean free path of the system are negligible.

The relaxation times, $\tau_{n}^{s}(\mathbf{k})$, which are related to the elapsed time between diffusive collisions with the granular layers are obtained from the mean free paths, $\ell^{s}$, given by Eq. (2) and from the semiclassical velocities as

$$
\tau_{n}^{s}(\mathbf{k})=\frac{\ell^{s}}{v_{z n}^{s}(\mathbf{k})},
$$

where $z$ denotes the direction perpendicular to the interfaces of the multilayer. But, when

$$
\ell^{s} \frac{\left|v_{n}^{s}(\mathbf{k})\right|}{\left|v_{z n}^{s}(\mathbf{k})\right|}>\lambda_{\mathrm{Ag}}
$$

expression (3) for the relaxation time is replaced by

$$
\tau_{\mathrm{Ag}}^{s}(\mathbf{k})=\frac{\lambda_{\mathrm{Ag}}}{\left|v_{n}^{s}(\mathbf{k})\right|}
$$

because $\lambda_{\mathrm{Ag}}$ should be the limiting diffusion length of these systems.

\section{METHOD OF CALCULATION AND RESULTS}

To obtain the band contribution to the conductivity we consider that successive granular layers are identical; thereafter each unit cell contains only one Co cluster. In spite of this, the smallest unit cell considered has 320 atoms while the largest one has 768 . We consider that all the clusters within a given sample are identical; this is a simplification of the model to make the calculations tractable, even if in the real samples the clusters have a size distribution responsible for the resistivity values observed, which are higher than the calculated ones. As we are interested in determining the electronic transport behavior around the coalescence threshold of the Co clusters, it is enough to study the percolation transition considering clusters with the same size. Because of the large number of atoms, in order to obtain the electronic structure, we use a tight binding Hamiltonian with $s, p$, and $d$ orbitals for the $\mathrm{Co}$ atoms and also for the $\mathrm{Ag}$ atoms which are nearest neighbors to the Co ones. The rest of the $\mathrm{Ag}$ atoms are described by only one $s$ orbital. We have checked this simplified approach comparing the magnetic moments of the Co atoms at the interfaces with a similar system which contains all the valence orbitals. Magnetism is derived from a Hubbard-like term solved in the unrestricted Hartree-Fock approximation and the hopping and on-site parameters have been taken from the corresponding bulk values to fit the electronic band structure obtained from $a b$ initio calculations as in Ref. 16. For the Hamiltonian hopping elements between $\mathrm{Co}$ and $\mathrm{Ag}$, averaged values are taken following Shiba's rule. ${ }^{17}$ For the calculation of the conductivity we use a mesh of $20000 k$ points homogeneously distributed in the first Brillouin zone. We normalize the conductivities to the case in which the Co atoms are replaced by Ag ones in order to be able to compare the results for the different systems.

We focus our attention on two aspects of CIP transport in the granular multilayers: (A) The evolution of the resistivity as a function of the degree of percolation of the magnetic clusters in the granular region and (B) the evolution of the resistivity as a function of the $\mathrm{Ag}$ spacer thickness for a given averaged cluster size and the transition from a multilayered to a granular alloy depending on this thickness. We compare, when available, with experimental results.

\section{A. Resistivity versus degree of percolation}

To study the evolution of the transport properties with growing Co clusters' size we consider multilayers with the same spacer thicknesses, $t_{\mathrm{Ag}}$, namely seven Ag monolayers. The Co clusters in the granular regions are embedded within three Ag layers. In Table I we give the composition of each 
TABLE I. Systems with different degrees of percolation. The second column gives the nominal composition of the unit cells, the silver spacers consist of seven layers with $32 \mathrm{Ag}$ atoms each. $x_{\mathrm{Co}}$ gives the relative $\mathrm{Co}$ concentrations in the granular regions of each system. By $P_{r}$, we mean the ratio of the interconnecting Co necks' cross sections to the corresponding Co clusters' cross sections, both taken perpendicular to the transport direction. $H_{r}$ is the ratio between the number of $\mathrm{Ag}$ atoms which are first nearest neighbors to the Co clusters' surface atoms to the total number of Ag atoms. The last two columns show the transverse mean free path, $\ell$, for both spin polarization; the values are given in units of $t_{\mathrm{Ag}}$.

\begin{tabular}{lccccccc}
\hline \hline & & System & $x_{\mathrm{Co}}$ & $P_{r}$ & $H_{r}$ & $\ell^{\uparrow}$ & $\ell^{\downarrow}$ \\
\hline Nonpercolated & (a) & $\left(\mathrm{Co}_{13} \mathrm{Ag}_{83}\right) / \mathrm{Ag}_{32 \times 7}$ & 0.14 & 0 & 0.14 & 13.55 & 2.46 \\
& (b) & $\left(\mathrm{Co}_{33} \mathrm{Ag}_{63}\right) / \mathrm{Ag}_{32 \times 7}$ & 0.34 & 0 & 0.27 & 6.67 & 1.74 \\
\hline Percolated & (c) & $\left(\mathrm{Co}_{43} \mathrm{Ag}_{53}\right) / \mathrm{Ag}_{32 \times 7}$ & 0.45 & 0.20 & 0.30 & 5.88 & 1.63 \\
& (d) & $\left(\mathrm{Co}_{59} \mathrm{Ag}_{37}\right) / \mathrm{Ag}_{32 \times 7}$ & 0.61 & 0.60 & 0.30 & 4.37 & 1.49 \\
\hline \hline
\end{tabular}

of the unit cells considered, together with the Co clusters relative concentrations, $x_{\mathrm{Co}}$. The percolated ratio, $P_{r}$, which measures the clusters' percolation degree, is given by the ratio of the projected surface area of the necks interconnecting the Co clusters perpendicularly to the transport direction ( $x$ direction) to the total projected clusters' area. In Table I, we also give in the fourth column the hybridization ratio, $H_{r}$, which is the ratio between the number of Ag atoms which are first nearest neighbors to the Co clusters' surface atoms with respect to the total number of $\mathrm{Ag}$ atoms. The systems are isotropic in the $x$ and $y$ directions. Systems (a) and (b) model granular multilayers with nonpercolated Co clusters $\left(P_{r}=0\right)$, while systems (c) and (d) are examples of percolated granular multilayers. These latter systems are formed by adding Co atoms to the clusters of system (b) in order to interconnect them. The Co necks of system (d) show a larger section than the ones of system (c), configuring two different degrees of percolation. In the last two columns in Table I, we also show the transverse component of the mean free path per spin, $\ell^{s}$, for the studied systems. We observe that $\ell^{s}$ decreases monotonously for both spin directions as the degree of percolation increases. This result agrees with the fact that the number of $d$ and of hybridized $s d$ states at the Fermi level increases with growing degree of percolation. We also observe that $\ell^{\downarrow}$ is systematically smaller than $\ell^{\uparrow}$, this is consistent with the fact that spin down electrons suffer a larger scattering than spin up ones due to a larger minority Co $d$ density of states.

In order to obtain just the band contribution to the resistivity we define a ratio which we call $\rho_{N}^{1}$. We calculate $\rho_{N}^{1}$ by setting $\tau$ equal to one in Eq. (1) and normalizing it with respect to a system with the same unit cell size but which contains only atoms of Ag type. Subindex $N$ indicates this normalization. In Fig. 1 we plot the ratio $\rho_{N}^{1}$ for the four systems indicated in Table I. As $\tau$ has been set equal to 1 in Eq. (1), the bands which cross the Fermi surface act just as conduction channels $(\mathrm{CChs})$ for which the semiclassical velocities are the only important quantity.

The fact that $\rho_{N}^{1}$ for system (a) is smaller than one, that is smaller than the $\rho_{N}^{1}$ corresponding to pure silver, indicates that Co $d$ bands contribute with a larger amount of CChs

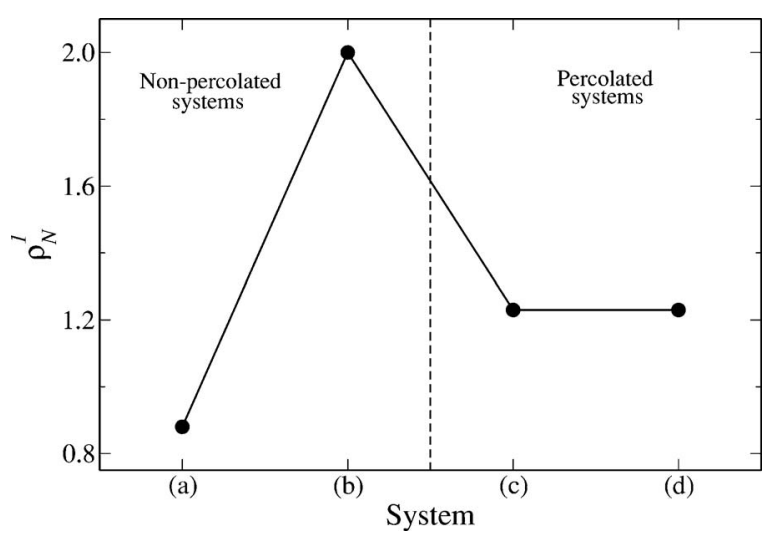

FIG. 1. Band contribution to the resistivity of the four systems studied normalized to the corresponding contribution of systems in which the Co atoms have been replaced by $\mathrm{Ag}$ ones. In $\rho_{N}^{1}$ the relaxation time, $\tau$, has been set equal to 1 .

than Ag. The largest value for $\rho_{N}^{1}$ is obtained for system (b) and this has to do with the fact that this system has a larger $\mathrm{Co} / \mathrm{Ag}$ interface ratio than system (a). System (c) is already topologically percolated and this is clearly seen in the decrease shown by $\rho_{N}^{1}$ when going from (b) to (c), remaining almost unchanged when going from (c) to (d). System (d) shows a larger degree of topological percolation than system (c). The data presented in Fig. 1 show an interplay between the number of $d$ channels of $\mathrm{Co}$, which leads to a decrease in the value of $\rho_{N}^{1}$ and an increasing degree of Co-Ag hybridization, $H_{r}$. As it can be seen, there exists a percolation threshold beyond which the resistivity value drops toward an asymptotic value.

In Fig. 2 we display the resistivity ratio $\rho_{N}$ for the earlier systems taking into account the band contribution and the scattering one. As mentioned earlier, in the calculation of the relaxation time we consider the scattering effects due to the localized $d$ states through a model mean free path given by Eq. (2). According to this, we calculate the resistivity $\rho_{N}$, which similarly as in the case of $\rho_{N}^{1}$, in each case is normalized with respect to systems that have the same number of atoms but all of them $\mathrm{Ag}$ atoms. It is interesting to note that the main contribution to the large value of $\rho_{N}$ for system (b) comes from Co-Ag hybridization at the interfaces. For systems (c) and (d), $\rho_{N}$ is close to 1 , which means that the

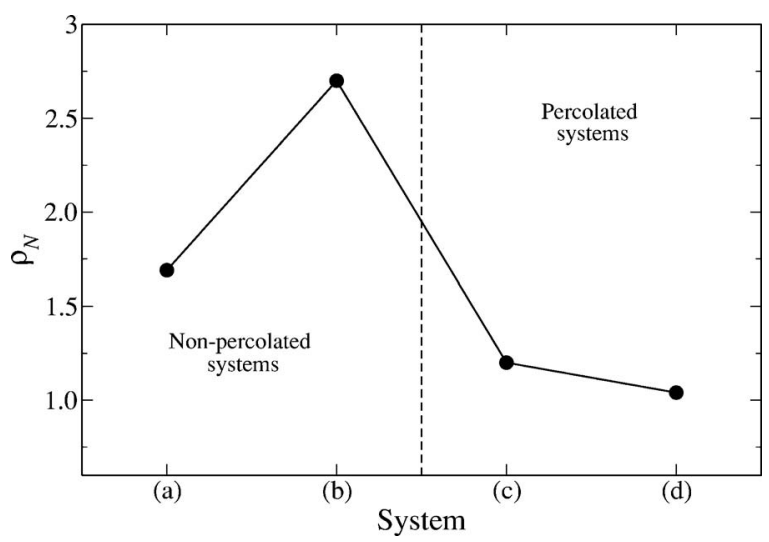

FIG. 2. Resistivity of the four systems studied normalized to systems in which the Co atoms have been replaced by Ag ones. 
resistivity has almost attained the Ag resistivity value. This result is in agreement with our previous experimental data, ${ }^{8}$ where we have shown that for $\mathrm{Co} / \mathrm{Ag}$ granular multilayers with percolated clusters the resistivity tends rapidly to the $\mathrm{Ag}$ value, even if the granular layers do not form complete continuous layers. Unfortunately, in the literature there are no systematic studies of the resistivity of these systems being the GMR the most studied quantity and, due to this, it is not easy to find experimental results to compare with.

As we stated in Sec. II, the disorder in the granular layers is taken into account in the calculation of $\ell$ but it is not considered in the band structure calculations (semiclassical velocities). Within our model, electrons find low resistivity paths which are not available in a real system with a random distribution of the shape and size of the cluster. This suggests that the resistivity values shown in Figs. 1 and 2 are underestimated even if the resistivity behavior as a function of the degree of percolation is not affected and reflects the experimental trends.

\section{B. Resistivity versus pure Ag layer thickness}

Instead of changing the amount of Co per unit cell we modify, in this case, the number of Ag layers in the metallic spacer region. We consider system (b) of the latter subsection because it is the one with the largest unpercolated Co clusters and, thereafter, the effect on the resistivity of an increasing number of $\mathrm{Ag}$ layers, should be more pronounced than in system (a).

Let us remember that the relaxation time contains a correction which takes into account that the electrons cannot travel over paths which are larger than the $\mathrm{Ag}$ mean free path. This contribution becomes now more important than in the previous section due to the fact that the systems have different $\mathrm{Ag}$ thicknesses and that the fraction of shortcircuited electrons depends on this thickness. We consider several values for $\lambda_{\mathrm{Ag}}$, given in units of the lattice constant of $\mathrm{Ag}$, in order to study this effect. For instance, when $\lambda_{\mathrm{Ag}}$ is large all the electrons travel large distances within $\mathrm{Ag}$ and, in spite of having a fraction of the electrons flowing almost parallel to the interfaces, a large amount of them will reach the Cols, thereafter, the short-circuit effect through Ag becomes less important. In the other extreme, for $\lambda_{\mathrm{Ag}}$ small enough, most of the electrons will short circuit through the Ag layers because the distance to be traveled between granular layers is almost equal or even larger than $\lambda_{\mathrm{Ag}}$.

In Fig. 3 we show $\rho_{N}$ as a function of Ag thickness (we also give the results as a function of Co relative concentration) for several values of $\lambda_{\mathrm{Ag}}$. For $\lambda_{\mathrm{Ag}}$ varying from 240 to 2400 in units of the bulk lattice constant we can observe three different regions depending on $x_{\mathrm{Co}}$ : (i) small variations of the resistivity ratio at high Co concentrations, (ii) a pronounced decrease in the resistivity at medium Co concentration and, (iii) an asymptotic approach of $\rho_{N}$ to the pure $\mathrm{Ag}$ resistivity value in the low Co concentration region. The behavior given in (i) reveals that in this concentration region and for this range of $\lambda_{\mathrm{Ag}}$ values, this length is much larger than $t_{\mathrm{Ag}}$, almost all electrons enter into the Col's and do not short circuit through the Ag layers. This result is reinforced

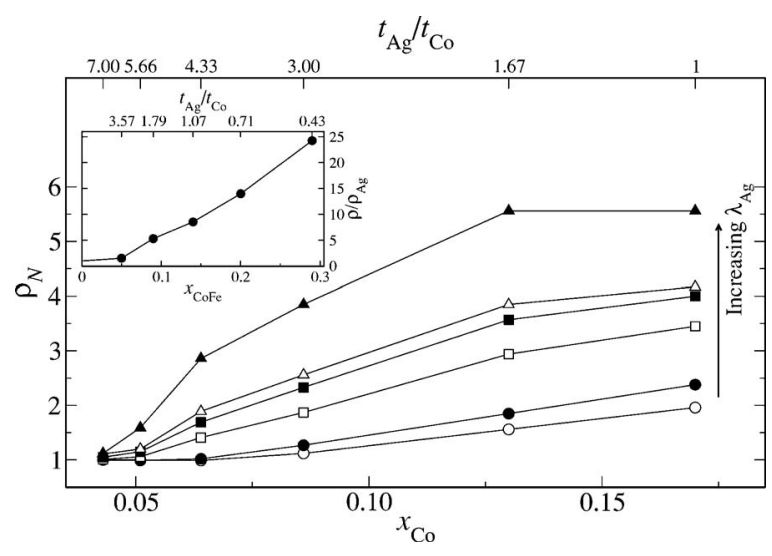

FIG. 3. Resistivity ratio as a function of Co concentration $\left(x_{\mathrm{Co}}\right)$ for the systems of Table II by considering several values for the Ag mean free path, $\lambda_{\mathrm{Ag}}$, which is given in units of the bulk Ag lattice constant and the symbols correspond to: $(\bigcirc) \lambda_{\mathrm{Ag}}=12 ;(\boldsymbol{O}) 24 ;(\square) 120 ;(\boldsymbol{\square}) 240 ;(\triangle)$ 360; and $(\boldsymbol{\Delta})$ 2400. In the inset, it is shown the experimental data extracted from Ref. 8.

by the fact that for increasing $\lambda_{\mathrm{Ag}}$ this effect is more pronounced reaching a flat behavior for the largest $\lambda_{\mathrm{Ag}}$ value used. At intermediate concentrations, that is in case (ii), $\rho_{N}$ shows a strong influence of scattering into localized states, but a fraction of the electrons start to flow only within Ag. Therefore, the resistivity decreases fast. For smaller Co concentrations, case (iii), the short-circuit effect through $\mathrm{Ag}$ is more important than scattering into localized states. It is important to see that the thicknesses covered by the different behaviors depend on the value of $\lambda_{\mathrm{Ag}}$, putting into evidence the interplay between the localized state scattering rate and $\lambda_{\mathrm{Ag}}$. The curves corresponding to $\lambda_{\mathrm{Ag}}=12,24$, and 120 just show the behavior described in (i) because short circuiting through Ag is always more important than scattering to localized states for these values of $\lambda_{\mathrm{Ag}}$.

It is interesting to compare the experimental curve, ${ }^{8}$ inset Fig. 3, with our results. It shows a similar behavior to the one presented by the curves corresponding to $\lambda_{\mathrm{Ag}}=12,24$. These values correspond to 49 and $98 \AA$, respectively, which are in good agreement with the order of the experimental mean free path value. The experimental resistivity ratios are larger than the ones calculated for higher Co concentrations. This is due to the random arrangement of the clusters and also to the CPP behavior that these kind of alloys should show when $t_{\mathrm{Ag}}$ is reduced, as we have shown in Ref. 8, and to the fact that we are not taking into account backscattering

TABLE II. Systems with varyng Ag spacer thickness. The first column gives the nominal composition of the unit cells, the silver spacers consist of a varying numbers of $\mathrm{Ag}$ layers with $32 \mathrm{Ag}$ atoms each. In this case, the Co concentration is given per formula unit. The last column gives the ratio of the Ag spacer thickness to the superlattice periodicity, for each system.

\begin{tabular}{ccc}
\hline \hline System & $x_{\mathrm{Co}}$ & $t_{\mathrm{Ag}} / t_{\mathrm{Co}}$ \\
\hline$\left(\mathrm{Co}_{33} \mathrm{Ag}_{63}\right) / \mathrm{Ag}_{32 \times 3}$ & 0.17 & 1.00 \\
$\left(\mathrm{Co}_{33} \mathrm{Ag}_{63}\right) / \mathrm{Ag}_{32 \times 5}$ & 0.13 & 1.67 \\
$\left(\mathrm{Co}_{33} \mathrm{Ag}_{63}\right) / \mathrm{Ag}_{32 \times 9}$ & 0.086 & 3.00 \\
$\left(\mathrm{Co}_{39} \mathrm{Ag}_{63}\right) / \mathrm{Ag}_{32 \times 13}$ & 0.064 & 4.33 \\
$\left(\mathrm{Co}_{39} \mathrm{Ag}_{63}\right) / \mathrm{Ag}_{32 \times 17}$ & 0.051 & 5.67 \\
$\left(\mathrm{Co}_{39} \mathrm{Ag}_{63}\right) / \mathrm{Ag}_{32 \times 21}$ & 0.043 & 7.00 \\
\hline \hline
\end{tabular}


effects important for the CPP-like contributions to transport within the present model. In spite of this, using rather academic values for $\lambda_{\mathrm{Ag}}$ allows us to follow the evolution of $\rho_{N}$ through the different transport regimes that a granular multilayer with nonpercolated clusters can present, taking into account only the CIP contribution. By increasing the $\lambda_{\mathrm{Ag}}$ value, we can model what would happen in system with $t_{\mathrm{Ag}}$ thinner than in our simulations. In those cases the CPP contribution to transport is larger and this allows us to determine the limits of our model. Namely, in Fig. 3 we show that for $\lambda_{\mathrm{Ag}}=2400$, one can detect three different transport regions for the CIP contribution, in the first one for thick metallic spacers (low Co concentration) the granular layers are decoupled, the electrons short circuit through the Ag layers and the resistivity tends fast toward the pure Ag value, almost independent of the $\lambda_{\mathrm{Ag}}$ value. In the second region, electrons see the interfaces of the multilayer and there is a growing degree of scattering to $d$ states, while in the last region one is beyond the CIP-granular limit, all electrons flow in a CPP-like regime, and backscattering effects should be certainly considered.

\section{CONCLUSIONS}

In this contribution we have studied the CIP transport behavior of $\mathrm{Ag} / \mathrm{Co}$ granular multilayers. On the one side we have looked for topological percolation effects of the Co clusters on the electrical resistivity of these multilayers and we have also studied the evolution of the resistivity as a function of Ag layer thickness in the case of unpercolated systems.

Having worked within the Boltzmann approximation, we have paid particular attention to the calculation of the relaxation time by proposing a model which takes into account scattering into the localized $d$ states of the clusters. The percolation effects have been considered through the features which emerge naturally out of the band structures, which have been obtained using a tight binding model Hamiltonian. Within this approach, the resistivity behavior is determined by the shape of an average Fermi surface and by the semiclassical velocities. We show that the percolative transition can already be detected through the evolution of the normalized resistivity with the only consideration of the band structure, as the CIP resistivity presents a percolation related drop. The model proposed for the relaxation time reproduces well the experimentally observed resistivity trends.

We have shown that in the limit of small Ag thicknesses the CIP contribution to the resistivity of unpercolated samples presents three transport regimes: one in which the granular layers are decoupled and the electrons short circuit through the Ag spacer, an intermediate regime, where both features, the multilayered as well as the granular one compete and in which an increasing fraction of electrons scatter into the $d$ states of the clusters, and a last regime in which the systems behave as granular alloys and the CIP contribution to the resistivity reaches its maximum value. Further studies must include backscattering processes in order to properly take into account the CPP contribution.

Summarizing, we have proposed a semiclassical treatment which can account for the resistivity behavior around the percolation limit of multilayered granular alloys and also for the crossover from a multilayered to a granular alloy transport behavior of these metallic systems. The obtained tendencies show a good overall agreement with the currently available experiments.

\section{ACKNOWLEDGMENTS}

The authors acknowledge Fundación Antorchas, UBACyT X-115 and PICT 03-10698 for partial support of this work. The authors acknowledge L. B. Steren for proposing to study these systems and S. Di Napoli for helpful discussions. The authors are members of CONICET.

${ }^{1}$ M. N. Baibich, J. M. Broto, A. Fert, F. Nguyen Van Dau, F. Petroff, P. Etienne, G. Creuzet, A. Friederich, and J. Chazelas, Phys. Rev. Lett. 61, 2472 (1988).

${ }^{2}$ A. E. Berkowitz, J. R. Mitchell, M. J. Carey, A. P. Young, S. Zhang, F. E. Spada, F. T. Parker, A. Hutten, and G. Thomas, Phys. Rev. Lett. 68, 3745 (1992); J. Q. Xiao, J. S. Jiang, and C. L. Chien, Phys. Rev. Lett. 68, 3749 (1992).

${ }^{3}$ S. Zhang and P. M. Levy, J. Appl. Phys. 73, 5315 (1993).

${ }^{4}$ L. Sheng, Z. D. Wang, D. Y. Xing, and J. X. Zhu, Phys. Rev. B 53, 8203 (1996).

${ }^{5}$ E. F. Ferrari, F. C. S. da Silva, and M. Knobel, Phys. Rev. B 59, 8412 (1999).

${ }^{6}$ P. Holody, L. B. Steren, R. Morel, A. Fert, R. Loloee, and P. A. Schroeder, Phys. Rev. B 50, 12999 (1994).

${ }^{7}$ E. A. M. van Alphen and W. J. M. de Jonge, Phys. Rev. B 51, 8182 (1995); Y. Luo, M. Moske, A. Kaeufler, T. Lorenz, and K. Samwer, J. Appl. Phys. 81, 4589 (1997); G. J. Bracho Rodríguez, L. G. Pereira, M. G. M. Miranda, A. B. Antunes, and M. N. Baibich, J. Magn. Magn. Mater. 214, 78 (2000).

${ }^{8}$ J. Milano, L. B. Steren, A. M. Llois, A. Butera, and J. Barnard, J. Appl. Phys. 96, 7392 (2004).

${ }^{9}$ F. Fettar, L. B. Steren, A. Barthélémy, R. Morel, A. Fert, J. A. Barnard, and J. D. Jarratt, J. Magn. Magn. Mater. 165, 316 (1997).

${ }^{10}$ N. Mott, Adv. Phys. 13, 325 (1964).

${ }^{11}$ E. H. Sondheimer, Adv. Phys. 1, 1 (1952).

${ }^{12}$ J. Milano, A. M. Llois, and L. B. Steren, Phys. Rev. B 66, 134405 (2002).

${ }^{13} \mathrm{~S}$. Datta, Electronic Transport in Mesoscopic Systems (Cambridge University Press, Cambridge, 1995), Chap. VII.

${ }^{14}$ J. M. Ziman, Electrons and Phonons (Oxford University Press, London, 1967), Chap. VII.

${ }^{15}$ P. Zahn, J. Binder, and I. Mertig, Phys. Rev. B 68, 100403(R) (2003).

${ }^{16}$ J. Guevara, A. M. Llois, and M. Weissmann, Phys. Rev. Lett. 81, 5306 (1998).

${ }^{17}$ H. Shiba, Prog. Theor. Phys. 46, 77 (1971). 